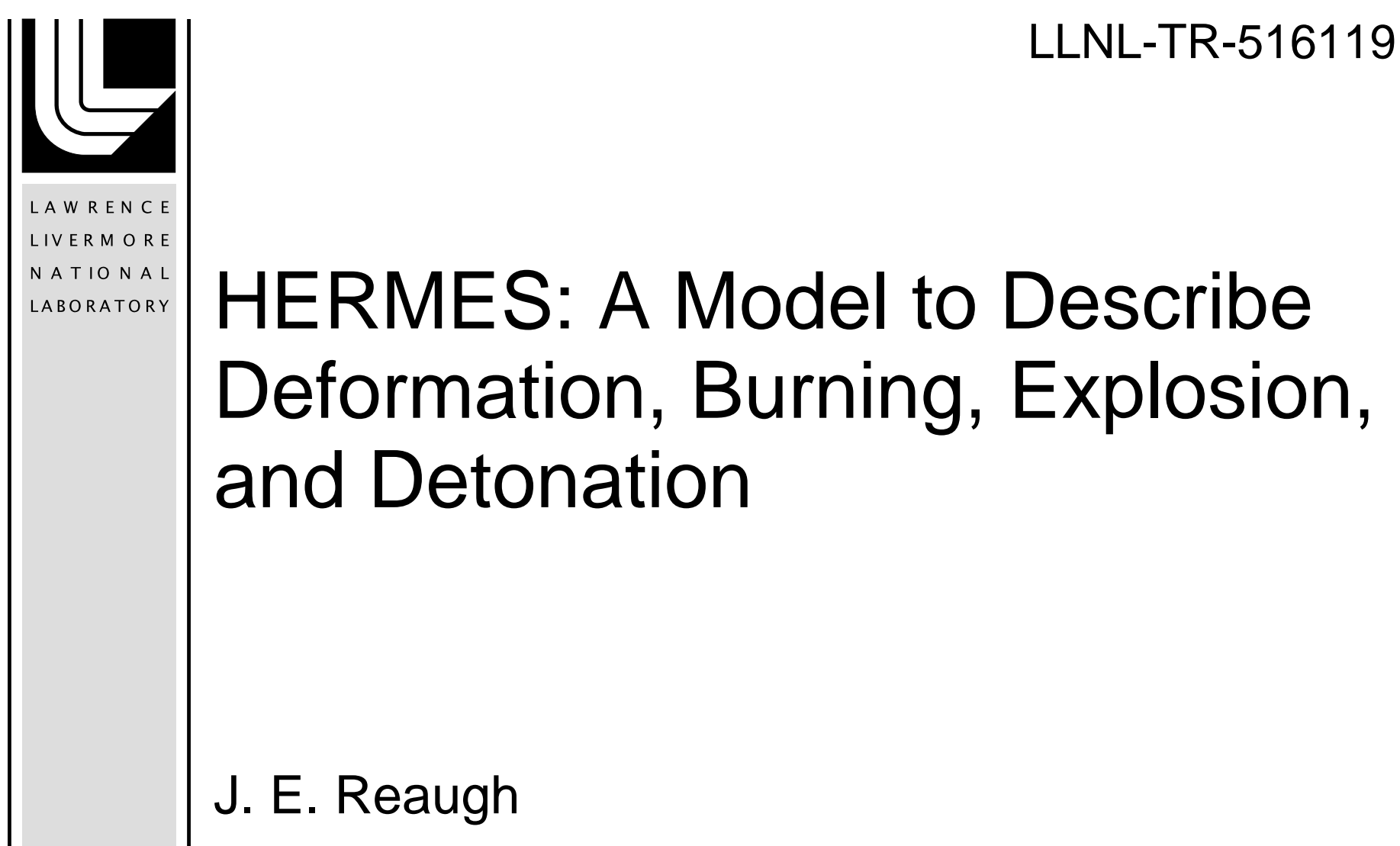

November 23, 2011 
This document was prepared as an account of work sponsored by an agency of the United States government. Neither the United States government nor Lawrence Livermore National Security, LLC, nor any of their employees makes any warranty, expressed or implied, or assumes any legal liability or responsibility for the accuracy, completeness, or usefulness of any information, apparatus, product, or process disclosed, or represents that its use would not infringe privately owned rights. Reference herein to any specific commercial product, process, or service by trade name, trademark, manufacturer, or otherwise does not necessarily constitute or imply its endorsement, recommendation, or favoring by the United States government or Lawrence Livermore National Security, LLC. The views and opinions of authors expressed herein do not necessarily state or reflect those of the United States government or Lawrence Livermore National Security, LLC, and shall not be used for advertising or product endorsement purposes.

This work performed under the auspices of the U.S. Department of Energy by Lawrence Livermore National Laboratory under Contract DE-AC52-07NA27344. 


\title{
HERMES: A model to describe deformation, burning, explosion, and detonation
}

\author{
John E. Reaugh \\ November 22, 2011
}

\section{Introduction}

HERMES (High Explosive Response to MEchanical Stimulus) was developed to fill the need for a model to describe an explosive response of the type described as BVR (Burn to Violent Response) or HEVR (High Explosive Violent Response). Characteristically this response leaves a substantial amount of explosive unconsumed, the time to reaction is long, and the peak pressure developed is low. In contrast, detonations characteristically consume all explosive present, the time to reaction is short, and peak pressures are high. However, most of the previous models to describe explosive response were models for detonation.

The earliest models to describe the response of explosives to mechanical stimulus in computer simulations were applied to intentional detonation (performance) of nearly ideal explosives [1-3]. In this case, an ideal explosive is one with a vanishingly small reaction zone. A detonation is supersonic with respect to the undetonated explosive (reactant). The reactant cannot respond to the pressure of the detonation before the detonation front arrives, so the precise compressibility of the reactant does not matter. Further, the mesh sizes that were practical for the computer resources then available were large with respect to the reaction zone. As a result, methods then used to model detonations, known as $\beta$-burn or program burn [4], were not intended to resolve the structure of the reaction zone. Instead, these methods spread the detonation front over a few finite-difference zones, in the same spirit that artificial viscosity [5] is used to spread the shock front in inert materials over a few finite-difference zones. These methods are still widely used when the structure of the reaction zone and the build-up to detonation are unimportant.

Later detonation models resolved the reaction zone $[6,7]$. These models were applied both to performance, particularly as it is affected by the size of the charge, and to situations in which the stimulus was less than that needed for reliable performance, whether as a result of accident, hazard, or a fault in the detonation train. These models describe the build-up of detonation from a shock stimulus. They are generally consistent with the mesoscale picture of ignition at many small defects in the plane of the shock front and the growth of the resulting hot-spots, leading to detonation in heterogeneous explosives such as plastic-bonded explosives (PBX). The models included terms for ignition, and also for the growth of reaction as tracked by the local mass fraction of product gas, $\lambda$.

The growth of reaction in such models incorporates a form factor that describes the change of surface area per unit volume (specific surface area) as the reaction progresses. For unimolecular crystalline-based explosives, the form factor is consistent with the mesoscale picture of a galaxy of hot spots burning outward and eventually interacting with each other. For composite explosives and propellants, where the fuel and oxidizer are segregated, the diffusion flame at the fuel-oxidizer interface can be interpreted with a 
different form factor that corresponds to grains burning inward from their surfaces [8]. The form factor influences the energy release rate, and the amount of energy released in the reaction zone. Since the $19^{\text {th }}$ century, gun and cannon propellants have used perforated geometric shapes that produce an increasing surface area as the propellant burns. This helps maintain the pressure as burning continues while the projectile travels down the barrel, which thereby increases the volume of the hot gas. Interior ballistics calculations use a geometric form factor to describe the changing surface area precisely. As a result, with a suitably modified form factor, detonation models can represent burning and explosion in damaged and broken reactant [9]. The disadvantage of such models in application to accidents is that the ignition term does not distinguish between a value of pressure that results from a shock, and the same pressure that results from a more gradual increase. This disagrees with experiments [10], where explosives were subjected to a gradual rise in pressure and did not exhibit reaction.

More recent models [11] do distinguish between slow pressure rises and shocks, and have had some success in the describing the response of explosives to single and multiple shocks, and the increase of shock sensitivity with porosity, at least over a limited range. The original formulation [12] is appropriate for sustained shocks, but further work is ongoing to describe the response to short pulses $[13,14]$.

The HERMES model [15-20] combines features from these prior models. It describes burning and explosion in damaged reactant, and also will develop a detonation if the gradual rise in pressure from burning steepens into a strong-enough shock. The shock strength needed for detonation in a fixed run distance decreases with increasing porosity. The model also incorporates an ignition criterion that can start burning from impact due to accidents and hazards that are too weak to cause shock-to-detonation directly. Because these impacts are weak, the build-up of reaction is slow and very sub-sonic. HERMES is therefore required to describe the compressibility and shear response of the reactant. The calculation of pressure equilibrium between the reactant and gas product includes both the compressibility of isolated reactant grains in a sea of products and the resistance of an interlocking structure of reactant grains to pore-collapse and shear deformation.

In this report we describe the features of the HERMES model in its present form. In addition, we highlight potential areas for future improvements, both in computational efficiency and more accurate treatment. Many of the features required for a complete model have been based on judgment in the absence of supporting experiments. When such experiments are performed, and the results analyzed, those features will likely require either refinement or complete reformulation. Applications of HERMES described here use the properties and experimental data for a UK developed explosive that is $91 \%$ HMX and $9 \%$ polymer/plasticizer by weight.

\section{Mechanical response of a mixture of reactant and product}

The HERMES model describes the constitutive response of a mixture of the reactant and product at a fixed composition. To the extent that product gas occupies connected porosity in the reactant matrix, the mixture should be represented in a multi-phase, multi-velocity formulation so that product gas can migrate from high-pressure regions to lower-pressure regions. For HERMES we have chosen instead to treat the mechanical response as multi-phase but single velocity. As a result, gas product is not transported 
between adjacent computational finite elements (zones). Ongoing research at LLNL [21] is investigating a complementary multi-phase multi-velocity approach to explosive response. Their research models burning and the propagation of an ignition front from a localized region throughout the porous volume.

\subsection{Calculation of pressure equilibrium}

The equation for pressure equilibrium is based on multi-phase, multi-velocity formulations used for DDT [22] and for calculations of interior ballistics [23]. When the reactant is sufficiently dilute, there is pressure equilibrium between the separated reactant particles, $s$, and the gas product, $g$. (See Fig. 1 left) If the reactant is not dilute, so that the reactant particles touch, then the matrix, $m$, of particles carries some of the external load. (Fig. 1 right) Experimental models using dynamic photoelasticity illustrate the stressbridging that can occur for the case with no gas pressure [24]. In general,

$$
P_{s}=P_{g}+P_{M} / \Phi_{s}
$$

where $P_{M}$ is the macroscopic matrix pressure exerted on a cross section that includes both reactant and gas product. $\Phi_{s}$ is the volume fraction of the reactant. Throughout, we assume that the volume fractions and area fractions are the same. The total pressure of the mixture is given by

$$
P=\Phi_{g} P_{g}+\Phi_{s} P_{s}=P_{g}+P_{M}
$$

where

$$
\Phi_{g}+\Phi_{s}=1
$$

The macroscopic matrix pressure is just the effective stress defined in soil and rock mechanics [25]. The shear strength of partially saturated soils and rocks has been observed to depend on the effective stress. We have formulated the shear strength in HERMES to be dependent on the macroscopic matrix pressure.

The matrix specific volume is given by

$$
v_{m}=v /(1-\lambda)
$$

where $\lambda$ is the mass fraction of product and $v$ is the specific volume of the mixture. It is unchanged by the partition of volume between the reactant and the gas product:

$$
v=\lambda v_{g}+(1-\lambda) v_{s}
$$

where $v_{s}$ and $v_{g}$ are the specific volumes of reactant and product. As a result, the macroscopic matrix pressure is unchanged during the iteration to pressure equilibrium [17]. We note that the volume fractions of product and reactant are just the result of dividing each term on the right hand side of Eq. 5 by $v$.

\subsection{Conservation of energy}

For the volume element as a whole, conservation of energy in the absence of heat transfer is given by

$$
d e=-(P+Q) d v+d w_{s}
$$

where $e$ is the specific internal energy, $P$ is the element pressure and $Q$ is artificial viscous pressure [5]. The term $w_{s}$ is the work done against the stress-deviator tensor of the 

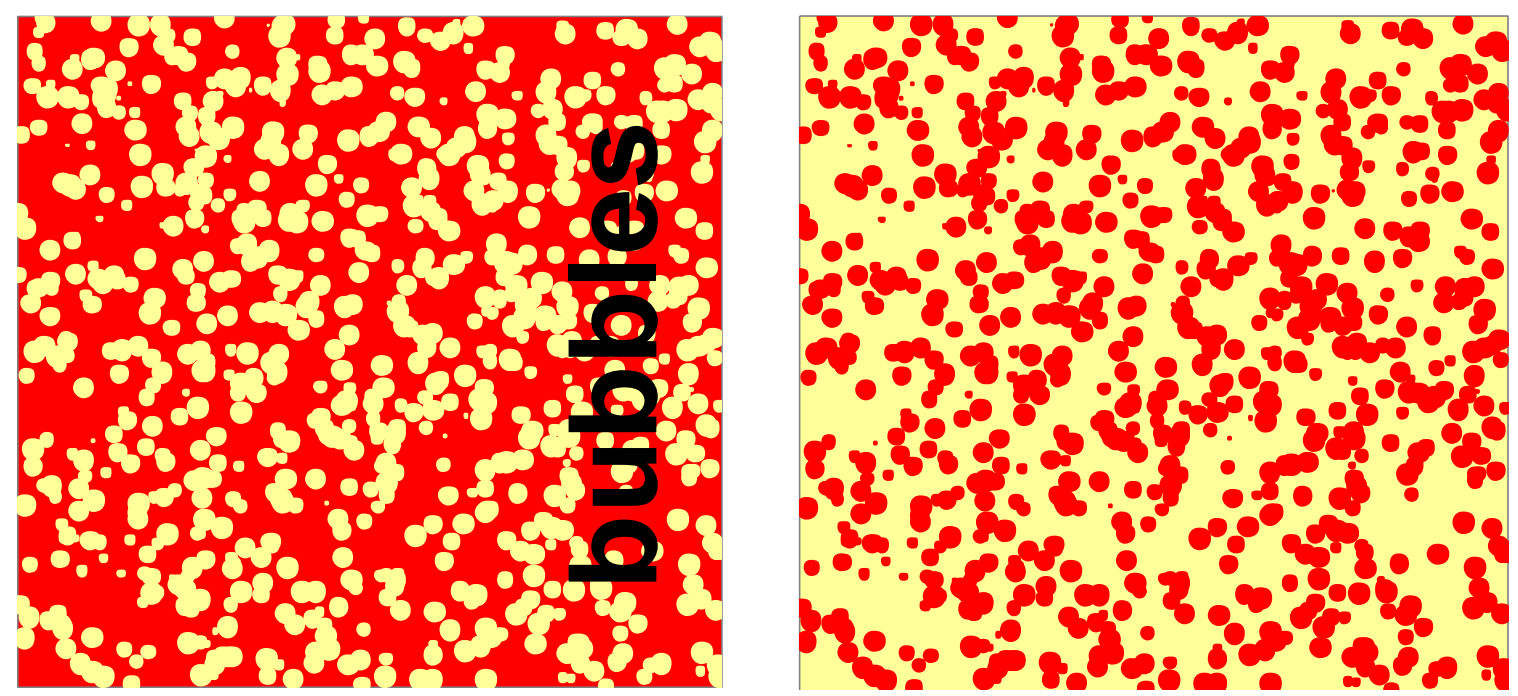

Figure 1. Illustration of dilute reactant ( $25 \%$ by volume, left) where the individual particles of reactant (yellow) are in pressure equilibrium with the surrounding gas product (red). When the reactant is not dilute (75\% reactant by volume, right), stress-bridging can occur from one side of the cross section to the other in a connected matrix of reactant.

matrix, including plastic work. The model must choose how to partition the energy among the constituents. We reject thermal equilibrium, because it is inconsistent with the mesoscale picture of hot spots growing into relatively cool reactant [26, 27]. Instead, we assume the reactant is on its adiabat [28-30], the matrix is on the new adiabat caused by shock loading, and the gas product picks up the remaining energy. When the reactant is too dilute to support a matrix stress, any shock heating goes to the more compressible gas.

The method described in [16] puts the gas on its adiabat and the solid (as distinct from the matrix) takes the energy left over. This is retained as an option in HERMES. None of these methods has proved to be entirely satisfactory. However, for burning and explosion applications, the pressures are low relative to a detonation, and the heating is relatively small. For this case the calculational results are not sensitive to the method chosen to partition the energy.

We noted in [18] that when the mass fraction of gas is small, the change in specific volume of the gas in response to a small total volume change can be quite large. Conservation of energy by a differential method becomes so inaccurate that the gas gets on an unphysical adiabat. We proposed a solution there, which requires calculating the entropy of each constituent. We have not implemented that solution for applications of the HERMES model.

\subsection{Calculation of gas product pressure}

We developed a tabular equation of state for the equilibrium gas products using Cheetah, a thermochemical equilibrium code developed at LLNL [31, 32]. Prior detonation models have used analytic equations of state for the gas products $[6,7,11]$. Those analytic equations are centered on the gas expansion adiabat from the Chapman-Jouget 
(CJ) detonation state. For the purpose to which they are intended, they are adequate. Our applications, however, include relatively low-pressure explosions, so that the gas is never near the $\mathrm{CJ}$ adiabat, unless a detonation develops later in time.

One shortcoming of our method is that thermochemical programs such as Cheetah are designed to be used only above the critical point. As a result, isotherms and adiabats near the critical point have a very low sound speed. Calculations (or table extrapolations) below the critical temperature exhibit van der Waals loops for both isotherms and adiabats. Such loops prevent the solution of pressure equilibrium by Newton methods (and also have imaginary sound speeds). Although one would not anticipate that applications of this model would explore low enough gas temperature for this issue to arise, the gas does occasionally find itself at low temperature. In our experience this was either as part of the phase space sampled during the iteration to pressure equilibrium, or due to the inaccuracy of differential energy conservation. We have not developed a robust solution to this issue.

\subsection{Calculation of solid pressure}

The reactant solid equation of state uses the Gruneisen form, but with the reference state taken to be the adiabat from room temperature and pressure, rather than the Hugoniot.

$$
P_{s}=P_{a d}(v)+\frac{\Gamma}{v}\left[e-e_{a d}(v)\right]
$$

where we have used

$$
P_{a d}(v)=A_{1} \mu+A_{2} \mu^{2}+A_{3} \mu^{3}
$$

where the excess compression is given by

$$
\mu=\frac{1}{\rho_{0} v}-1
$$

The specific energy density on the adiabat, $e_{a d}$, is given by

where the relative volume

$$
\begin{gathered}
\rho_{0} e_{a d}(v)=A_{1}[V-\ln (V)-1]+A_{2}\left[\frac{1}{V}-V+2 \ln (V)\right]+ \\
A_{3}\left[V-\frac{3}{V}+\frac{1}{2 V^{2}}-3 \ln (V)+\frac{3}{2}\right]
\end{gathered}
$$

$$
V=\rho_{0} v .
$$

We represent the Gruneisen by a low-order polynomial

$$
\frac{\Gamma}{v}=\rho_{0}\left(B_{0}+B_{1} \mu+B_{2} \mu^{2}\right)
$$

Throughout, $\rho_{0}$ is the reference state mass density of the (non-porous) solid reactant.

\subsection{Calculation of the matrix pressure}

The matrix compaction is based on the $P$ - $\alpha$ model described by Herrmann [33]. The fundamental premise of that model is that if the solid equation of state has the form

$$
P_{s}=f\left(v_{s}\right)
$$


then the porous equation of state has the form

$$
P_{p}=f\left(\frac{v}{\alpha}\right)
$$

where Herrmann's porosity measure, $\alpha$, was given by

$$
\alpha=\frac{v}{v_{s}} \text {. }
$$

However, in his formulation the mass fraction of gas product is always zero. In our generalization, we replace the specific volume of the mixture, $v$, (Eqs. 14 and 15) by the matrix specific volume, $v_{m}$.

$$
v_{m}=\frac{v}{1-\lambda}
$$

In that way, the porosity, $\phi$, which is identical to the volume fraction of product, $\Phi_{g}$, is

$$
\varphi=\frac{\lambda v_{g}}{v}=\Phi_{g}=\frac{v-(1-\lambda) v_{s}}{v}=1-\frac{v_{s}}{v_{m}}=1-\frac{1}{\alpha} .
$$

A crush pressure limits the pressure of the matrix,

$$
P_{c}=g\left(\alpha, \alpha_{0}\right)
$$

where $\alpha$ is the current porosity measure and $\alpha_{0}$ is its original value. If the unload-reload pressure $P_{p}$ exceeds the crush pressure, a new value of $\alpha$ is calculated to make them equal at the current specific volume.

We modified Herrmann's premise in two ways. First, we assume that the unloadreload and crush curves are principal adiabats. In Herrmann's examples the reference curves were taken to be the normal Hugoniot of the solid. In other applications [34] the reference curves have been quasi-static (isothermal) unloading and crush curves. Second, we reduce the stiffness of the unload-reload modulus by a linear function of porosity,

$$
f_{r}=f_{r 1}+\left(1-f_{r 1}\right) \frac{\alpha_{x}-\alpha}{\alpha_{x}-1}
$$

Here $\alpha_{x}$ is the maximum porosity that can withstand compressive stress and $f_{r l}$ is a parameter. In our work $\alpha_{x}$ is taken to be about 1.6 and $f_{r l}$ is taken to be about 0.2. The unload-reload curve is then given by

$$
P_{p}=f_{r} f\left(\frac{v_{m}}{\alpha}\right)
$$

A reduction of the stiffness of the unload-reload curve is often observed experimentally. In addition, we found that calculations using a different porous crush algorithm [35] were improved when such a stiffness reduction was included. We use the solid reference adiabat (Eq. 7) as the basis $f\left(v_{s} / \alpha\right)$ to calculate the unload-reload adiabat.

\subsection{Calculation of the flow stress}

The flow stress of a PBX depends on the initial temperature, strain, strain-rate, porosity, and pressure. The polymeric binder itself is temperature, pressure, and strainrate dependent. Above the glass-transition temperature, a decrease in the initial temperature results in a strength that obtains at the original temperature but higher strain rate. Typically, $10{ }^{\circ} \mathrm{C}$ corresponds to a decade of strain rate. For HMX-based explosives, the explosive crystals are harder than the binder, and the flow stress of this highly-filled 
composite is pressure dependent in the same way that rocks, soils, and concretes are pressure-dependent. For TATB based explosives formulated with high-density binders, the binder is harder than the crystals, at least at the temperatures used to polish specimens for microscopic evaluation. As a result, our formulation may need substantial alteration when applied to such explosive formulations at low temperature or high strain rate. We have based our model for strength on a model developed for concrete under dynamic load, which was similarly strain, strain-rate, porosity, and pressure dependent $[36,37]$.

Our algorithm calculates the strength for a given (trial) plastic strain-rate. The plastic strain increment resulting from that strength is divided by the time-step and compared to the trial strain-rate. We solve for a consistent strain-rate and strength by iteration. We found that bisection was the only iteration method robust enough to find a solution in all cases. It requires about 20 iterations to converge. A more efficient solution method would be advantageous.

\subsection{Calculation of the flow stress at constant strain rate}

The pressure- and strain- dependent strength [18] is given by

$$
Y=Y_{i} f_{e}(1-\Omega)+Y_{r} \Omega \text {. }
$$

Where $\Omega$ is a damage measure that varies between zero and one, $f_{e}$ is a strain hardening measure that has the maximum value of one, $Y_{i}$ is the strength of the undamaged (intact) reactant, and $Y_{r}$ is the (residual) strength of the fully damaged material. The damage parameter $\Omega$, is given by

$$
\Omega=\frac{D \max \left(0, \varphi-\varphi_{c r}\right)}{1+D \max \left(0, \varphi-\varphi_{c r}\right)}
$$

where $\varphi$ is the porosity, and where $D$ and $\varphi_{c r}$ are parameters. The strain hardening term $f_{e}$ is calculated by

$$
f_{e}=\delta+C(1-\delta)
$$

where $\mathrm{C}$ is a parameter between zero and one. The strain hardening measure $\delta$, which varies between zero and one, is calculated by

$$
\delta=\frac{\varepsilon_{p}}{\varepsilon_{p}+\varepsilon_{h}} .
$$

where $\varepsilon_{h}$, is a parameter and $\varepsilon_{p}$ is the plastic strain. The strength of fully damaged material (residual strength) is given by

$$
Y_{r}=R Y_{0}\left(\frac{A_{r}}{Y_{0}}+B_{r} \frac{p}{R Y_{0}}\right)
$$

where $A_{r}, B_{r}$, and $Y_{0}$ are parameters. The residual strength is constrained not to exceed the intact strength. For geologic materials, the parameter $A_{r}$ is taken to be zero to represent cohesionless soils and broken rock [36]. Here we let the parameter $A_{r}$ be larger than zero to represent the observed rate-dependent residual strength of explosive at (nearly) zero pressure. The strain-rate parameter $R$ is calculated by

$$
R=\left(1+\frac{\dot{\varepsilon}_{p}}{\dot{\varepsilon}_{0}}\right)^{e p}
$$


where $\dot{\varepsilon}_{p}$ is the plastic strain rate, and where ep and $\dot{\varepsilon}_{0}$ are parameters. We note that this form, proposed in [36], has the disadvantage of being unbounded at high strain rates. The experimental data typically available from Split Hopkinson Pressure Bar apparatus (SHPB) are limited to a strain rate of a few thousand per second. Dynamic tests, even at velocity $<30 \mathrm{~m} / \mathrm{s}$ can lead to local strain rates more than ten times the SHPB limit. In plane shocks, the strain rates are a few hundred times that limit. As a result, the extrapolated rate-dependent strength can become larger than is plausible. An alternative form retains the functional dependence fit to the SHPB data, but approaches a finite limit, $R_{\max }$, as the strain-rate increases further.

$$
R=1+\frac{R_{\max }-1}{2}\left(1+\tanh \left\{\left[\ln \left(\dot{\varepsilon}_{p} / \dot{\varepsilon}_{0}\right)-\ln \left(\varepsilon_{1}\right)\right] / c\right\}\right)
$$

where $\varepsilon_{1}$ and $c$ are parameters. We have not yet implemented this option.

The strength of the intact material is given by

$$
Y_{i}=R Y_{0}\left[\sqrt{s+m \frac{p}{R Y_{0}}+(m / 6)^{2}}-(m / 6)\right]
$$

for $p \geq 0$. We let $p$ stand for the macroscopic matrix pressure. Here $s$ and $m$ are parameters and $s$ generally takes the value one. This functional form suggested in [36] has the advantage over a previous form [17] in that it is explicit, rather than requiring solution by iteration. A disadvantage shared by the previous form is that it is an unlimited function of pressure. Where the pressure dependence is determined by quasi-static triax tests, the lateral confinement, and hence the pressure is limited to a few hundred MPa. The pressure developed in strong shocks is essentially unlimited, and so the strength using Eq. 28 is also unlimited. One expedient is to limit the expression in brackets to be a multiple somewhat larger than that for the largest pressure tested. An alternative, also used in rock mechanics is to use an exponential approach to an upper limit. This has the advantage of a smoothly varying derivative, which is required by Newton iteration schemes. We have occasionally included a limit on the pressure dependence, but have not implemented such a limit in general. For $p<0$, a straight line interpolation between the uniaxial tensile strength, $Y_{t}$, and $Y_{i}(0)$ is extrapolated to the pressure intercept at zero strength.

$$
Y_{i}(p<0)=Y_{i}(0)+\frac{3\left[Y_{i}(0)-Y_{t}\right]}{Y_{t}} p
$$

where $Y_{t}$ is the equal to $b_{t e n} Y_{0}$ where $b_{t e n}$ is the ratio of uniaxial tensile strength to uniaxial compressive strength. For rocks the ratio typically takes the value 0.1 . The floor value of $Y_{i}$ is zero. $Y_{i}$ is the flow stress when two of the three principal stresses are equal and are less compressive than the third. If the Mohr-Coulomb modification is desired, the flow stress is further modified by the appropriate factor given in section 3.3 .

The present calculation of the pressure dependence of strength is explicit in pressure, and so does not require iteration as did the previous version. As a result, simulations of the Steven test using the new calculation were observed to require only $60 \%$ of the computer time needed by the previous pressure dependent calculation. In addition, the use of residual strength improves the fit to the experimental data. Figs. 2 and 3 show the experimental data, $[38,39]$ along with the fit developed with the previous strength model [17], which was an implicit function of pressure. The triax tests (Fig. 2) are quasistatic 


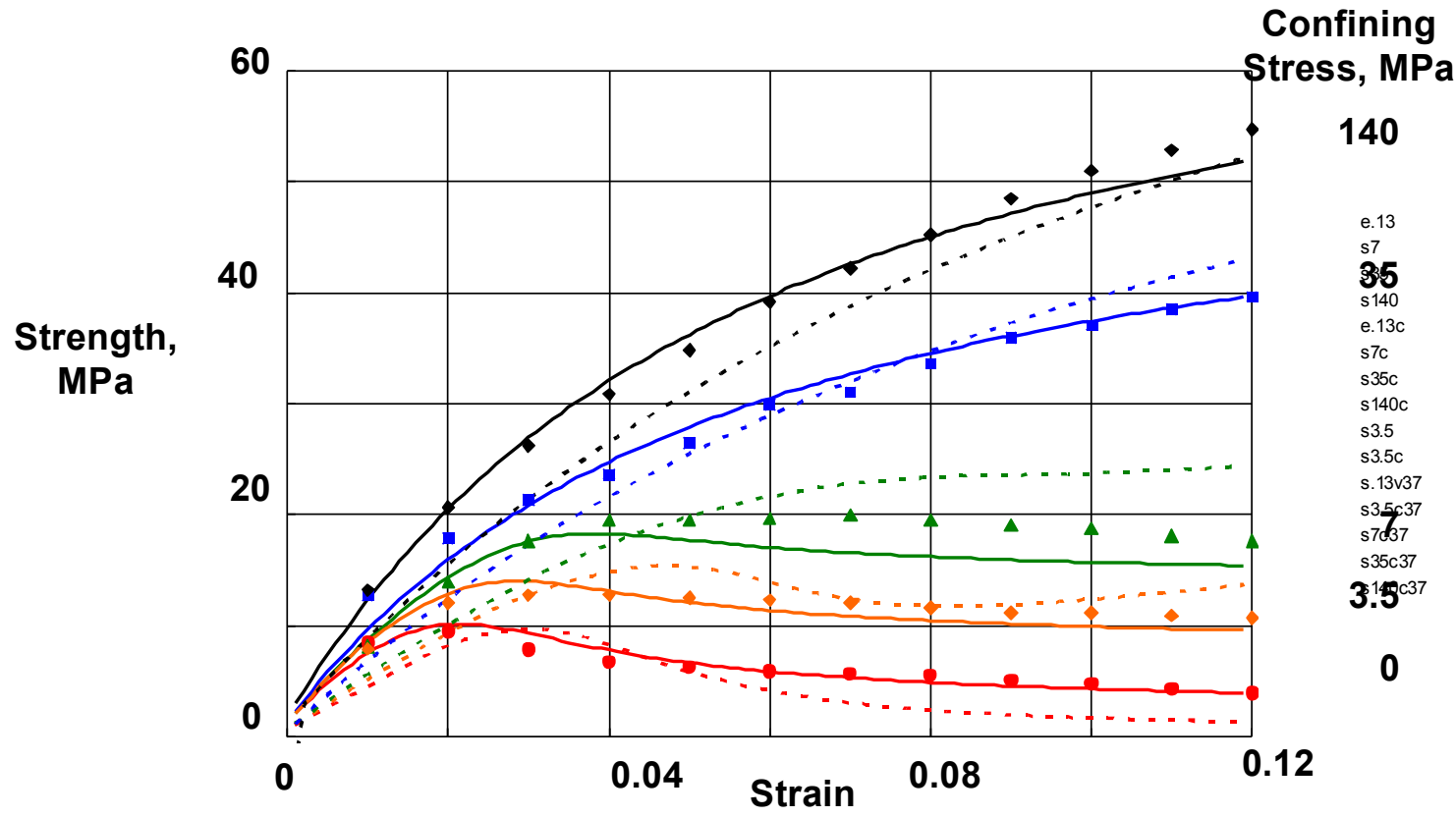

Figure 2. Model (solid) and previous model (dash) fits to quasistatic triax test data (symbols) [39] for various confining stresses.

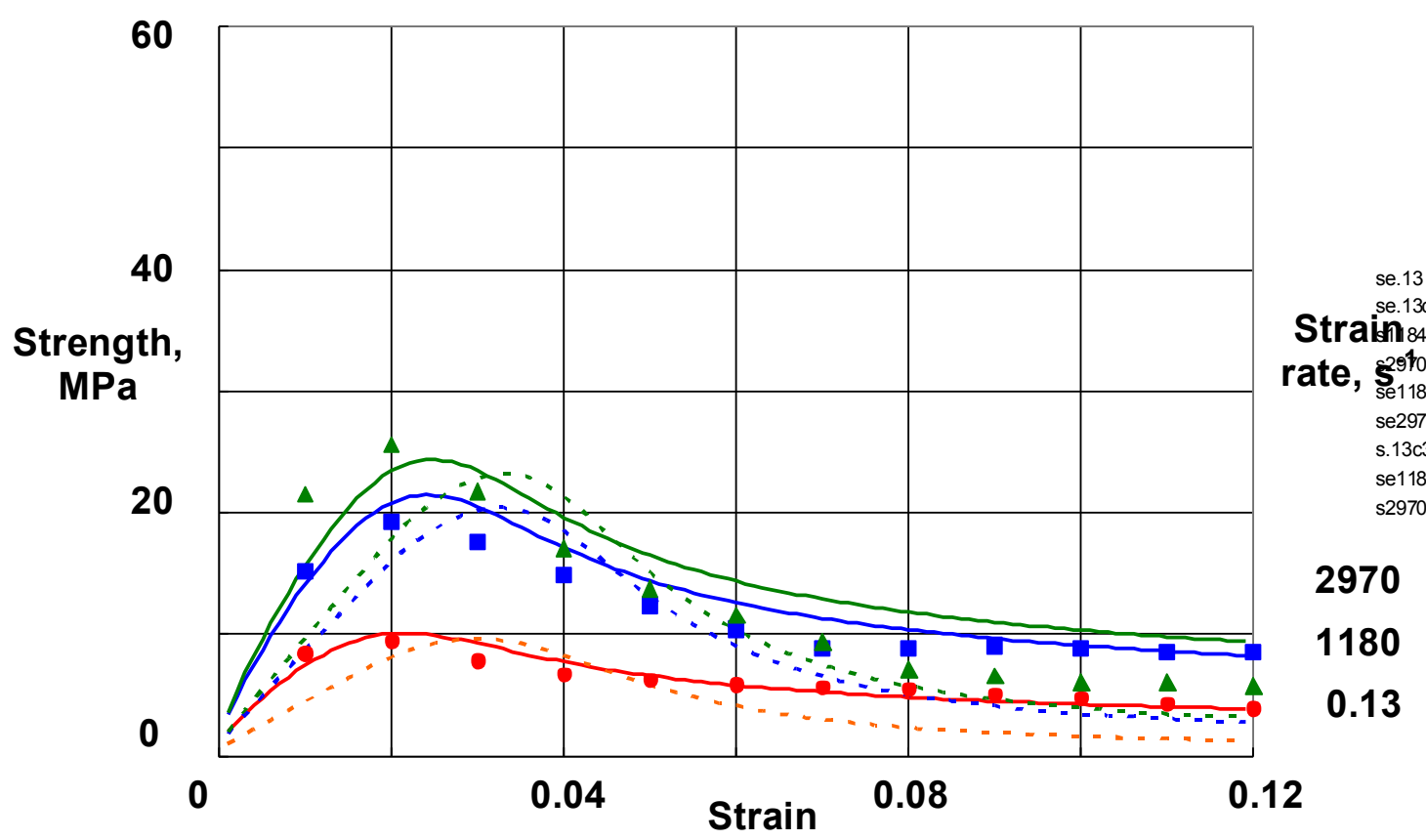

Figure 3. Model (solid) and previous model (dash) fit to dynamic split Hopkinson bar and quasistatic uniaxial compression data (symbols) [38] at various strain rates.

tests performed by first applying the confining stress as an isostatic load to a cylindrical sample, and then applying additional axial force, while maintaining the confining stress. Data from the split Hopkinson pressure bar are dynamic, but the confining stress is zero. 
The model treats combined strain-rate and pressure using Eq. 28 . At present there are no high strain-rate data with high confining stress for this explosive that can be used to test the model predictions.

\subsection{The Mohr-Coulomb modification}

For metals and clays, the von Mises criterion for strength is widely used. In principal stress space, with axes $\left(\sigma_{1}, \sigma_{2}, \sigma_{3}\right)$, the von Mises criterion is a circular cylinder whose axis is the principal diagonal. A cross-section normal to that cylinder (at constant pressure) is a circle, whose radius is the equivalent stress. See Figure 4. Although experiments to probe the yield surface are difficult, they have been done in a few instances, and for metals and clays, the yield surface can be nearly circular. Some tests with an aluminum alloy [40] have shown that even for metals, the equivalent strength in shear may not lie on the circle scaled to compression or tension. We are ignoring here such effects as kinematic hardening, whereby the circle does not expand with increasing plastic strain, but rather translates in the direction of straining.

In contrast, for geologic and granular materials, the Mohr-Coulomb criterion is widely used [41]. The condition for that criterion is

$$
\frac{\sigma_{c}-\sigma_{t}}{2}=f_{1}\left(\frac{\sigma_{c}+\sigma_{t}}{2}\right),
$$

where $\sigma_{c}$ is the most compressive principal stress and $\sigma_{t}$ is the most tensile principal stress. In this formulation, compressive stresses are positive. Most US and UK hydrodynamics codes follow the convention described by Wilkins [4, 42] so that the principal stresses and stress deviators are positive in tension, and pressure is positive in compression. In terms of the principal stress deviators ordered algebraically, we have

$$
s_{1} \geq s_{2} \geq s_{3}, \quad s_{1}+s_{2}+s_{3}=0 \text {. }
$$

The Mohr-Coulomb criterion is rewritten for that convention as

$$
\frac{s_{1}-S_{3}}{2}=A+B\left(p-\frac{s_{1}+s_{3}}{2}\right),
$$

where we have made the general functional dependence a (locally) linear one.

For our explosive, the data for pressure, strain-, and strain-rate dependent flow strength were taken in either uniaxial or triaxial compression. In uniaxial compression, the lateral stress is zero, whereas in triaxial compression, the lateral stress is compressive. In either case, two of the three principal stresses are equal and less compressive than the third (axial) component. It is therefore convenient to make the basis for the model the equivalent stress in compression, so that

$$
Y_{c}=f_{2}(p) \text {. }
$$

It is convenient to express other states of stress by the parameter $\xi$ where

$$
s_{2}=\xi s_{1} \text {. }
$$

Since we use the radial return method $[4,42]$ for reducing the stress tensor from the elastic trial state to the yield surface, the parameter, $\xi$ can be evaluated from the elastic trial state before the flow stress is calculated. For uniaxial compression, $\xi=1$. For uniaxial tension, $\xi=-1 / 2$. In shear, $\xi=0$. In uniaxial compression, Eq. 32 can be written

$$
\frac{3 s_{1}}{2}=A+B\left(p+s_{1} / 2\right) \text {. }
$$


We then evaluate the yield stress in compression as

$$
Y_{c}=\sqrt{\frac{3}{2}\left(s_{1}^{2}+s_{2}^{2}+s_{3}^{2}\right)}=3 s_{1}
$$

So that Eq. 32 becomes

$$
\frac{Y_{c}}{2}=A+B\left(p+\frac{Y_{c}}{6}\right) \text {. }
$$

We evaluate B from Eq. 37 by calculating the tangent to the yield surface (Eq. 33).

$$
B=\frac{f_{2}^{\prime}}{2+f_{2}^{\prime} / 3},
$$

where $f_{2}^{\prime}$ is the pressure derivative of $f_{2}$. At present we are using an expedient function $A_{r} \exp \left(-p / Y_{0}\right)$ for the pressure derivative. We found that the discontinuous change in the pressure derivative when the residual strength (Eq. 25) is limited to be no larger than the intact strength (Eq. 28) led to a discontinuous change in the slope, $B$, and a discontinuous change to the calculated strength. This prevented convergence. A more satisfactory solution would be to force the residual strength to approach the intact strength gradually as a function of pressure. This has not yet been implemented.

We evaluate Eq. 32 for the general value of $\xi$ to obtain

$$
(1+\xi / 2) s_{1}=A+B p+B \xi s_{1} / 2,
$$

We use Eq. 37 to evaluate $(A+B p)$ in terms of $Y_{c}$, and note that

$$
Y_{\xi}=\sqrt{3\left(1+\xi+\xi^{2}\right)} s_{1} .
$$

In terms of the compressive yield stress,

$$
Y_{\xi}=\frac{\sqrt{3\left(1+\xi+\xi^{2}\right)}(1-B / 3)}{2+(1-B) \xi} Y_{c} .
$$

The intersection of the yield surface with the plane perpendicular to the main diagonal or pressure axis, $\sigma_{1}=\sigma_{2}=\sigma_{3}$, is shown in Fig. 4 for three values of $f_{2}^{\prime}$. The extreme value of three is the largest possible value, and corresponds to a friction angle of $90^{\circ}$ where $B$ takes the value one. The slope of one $(B=0.43)$ is typical of rocks and soils. The value zero corresponds to no pressure dependence, and for that case the yield surface is equivalent to the Tresca condition [40]. The importance for our work is that the equivalent strength in pure shear is reduced relative to that in compression between the values 0.866 for no pressure dependence and 0.577 for the pressure derivative equal to three.

We have implemented this model as a user option and applied it to the calculation of the residual dent in the UK modification of the Steven test [19]. At $70 \mathrm{~m} / \mathrm{s}$, the (extrapolated) dent obtained in the test is about $12 \mathrm{~mm}$. The standard model uses the von Mises "circle." The calculation with the present model (Figs. 2, 3) and with Teflon strength taken to be $30 \mathrm{MPa}$ [43], results in an $11.7 \mathrm{~mm}$ dent. When the Mohr-Coulomb option is used, the calculated dent is $12.2 \mathrm{~mm}$. The calculated dents with and without the Mohr-Coulomb option are shown in Figure 5. Previously reported calculations of the dent [19] with the old fit showed a similar increase when the strength was reduced. Reducing the strength of the steel cover plate from 0.5 to $0.3 \mathrm{GPa}$ increased the dent by $0.5 \mathrm{~mm}$. Reducing the maximum strength of the $\mathrm{HE}$ from 0.3 to $0.15 \mathrm{GPa}$ increased the dent by $0.7 \mathrm{~mm}$. 


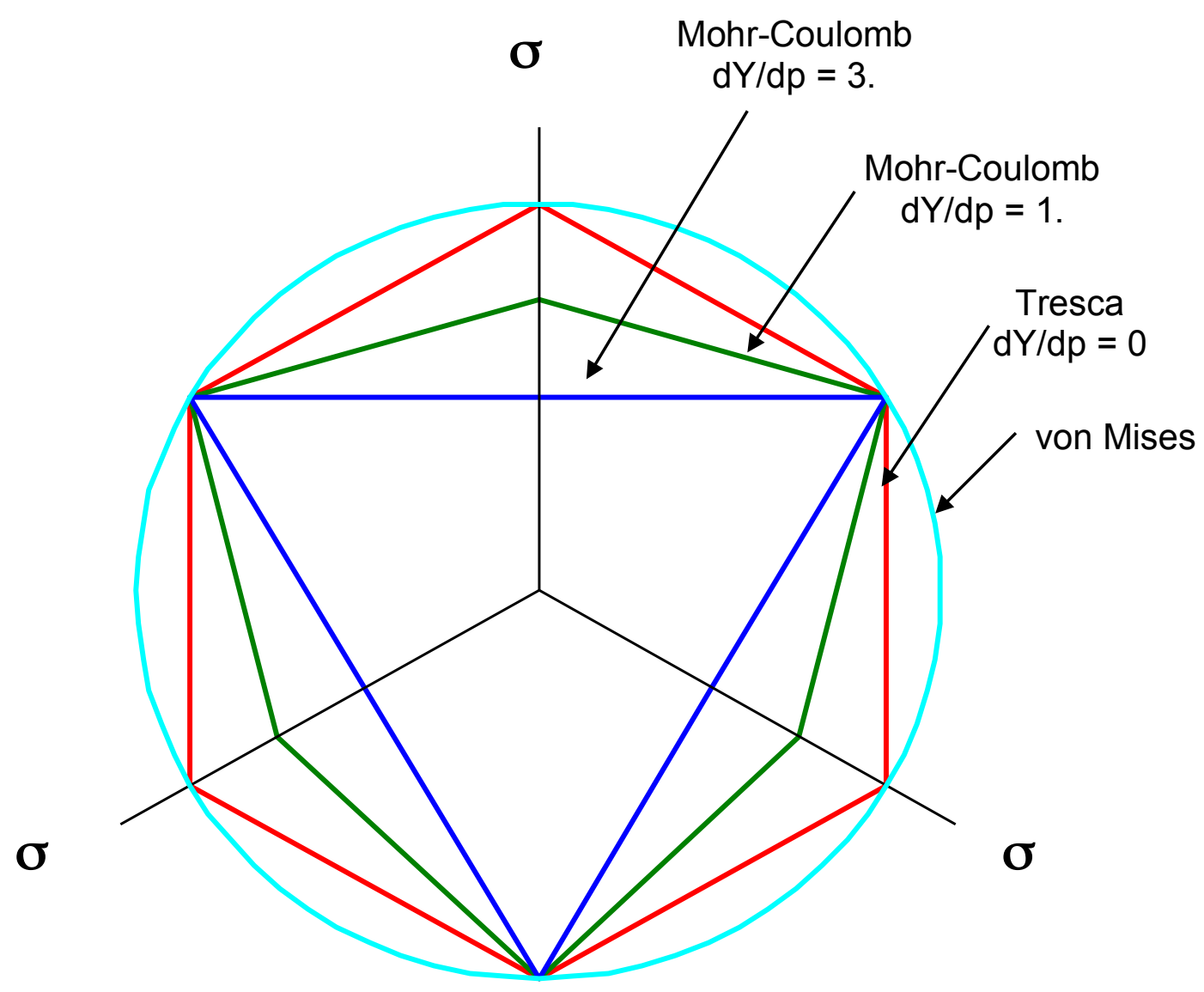

Figure 4. Intersection of the yield surface with a plane normal to the pressure axis. All surfaces are normalized to have the same value in triaxial compression. The three axes (thin black lines) are the three principal stresses. In this figure, the principal stresses are not ordered.

A modification of the triax test might be used to determine the suitability of the Mohr Coulomb model. After the application of isostatic stress, the axial load could, in principle, be reduced, rather than increased. This loading path is triaxial "tension" although all principal stresses are compressive. In Fig. 4, triaxial tension obtains on the corners of the yield surface that (apparently) intercept the three principal stress axes. The calculated and measured strengths could then be compared at several different confining pressures.

\section{Calculation of the increase of gas product}

An important use of the HERMES model is to quantify the amount of reactant that is converted to gas product as a function of time. The perceived violence of the event depends on both the amount of product and the time over which it is formed. For a given violence metric there is associated a characteristic time. If the product mass is developed more quickly than that, the violence measure is about the same as that produced by a detonation of the same mass of explosive. If the time to burn takes much longer than that, the violence measure is much reduced over that produced by a detonation. We quantified 

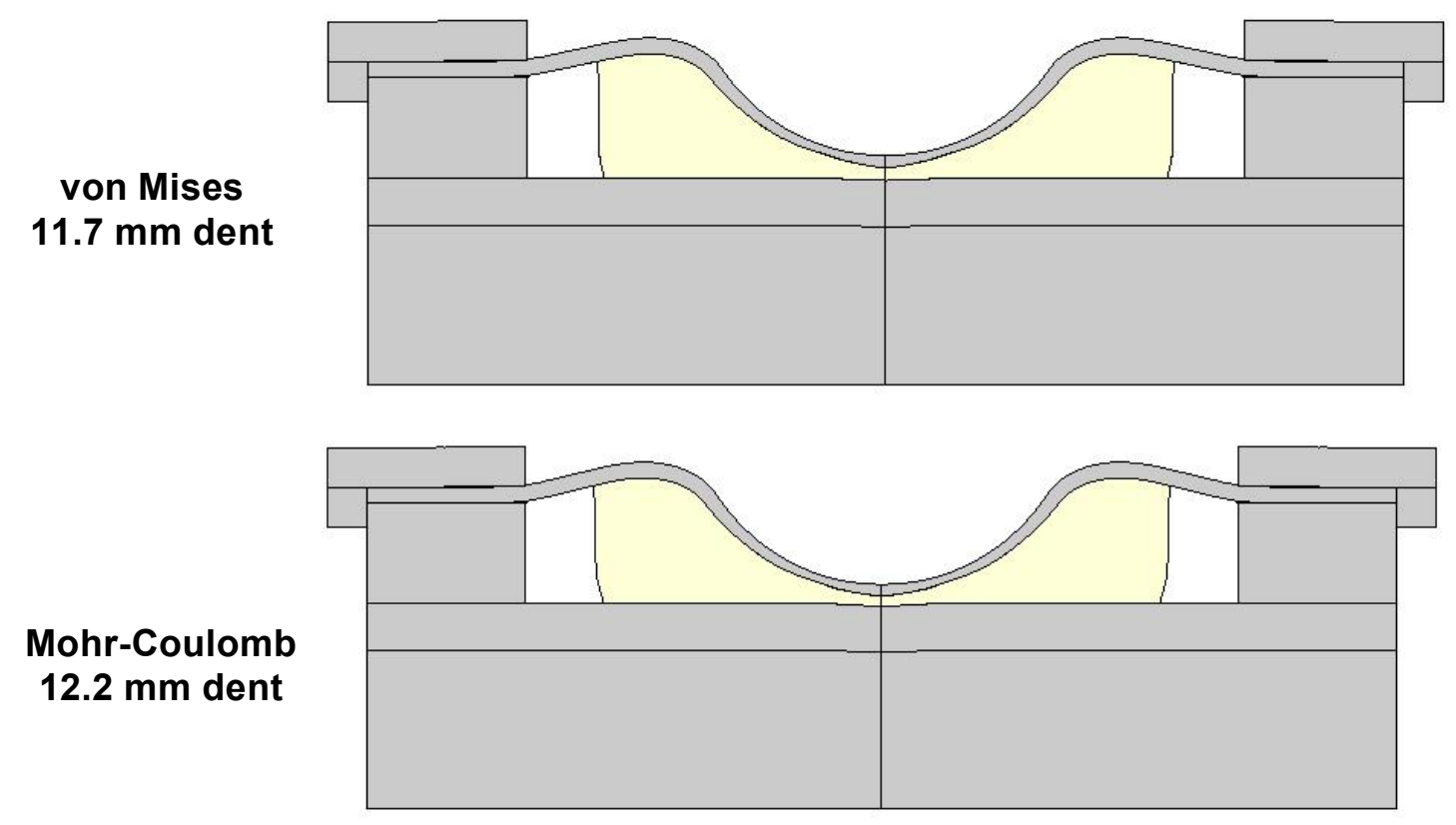

Figure 5. Calculated dents in the UK Steven test without (top) and with (bottom) the Mohr-Coulomb option.

this effect when air blast is used as the violence measure [44]. A similar effect, although with different characteristic times, also holds for the response of the surrounding confinement, whether measured by crater volume or shrapnel velocity.

In HERMES, there are separate submodels for ignition from a mechanical insult that does not produce shocks, the propagation of an ignition front through a mass of damaged material, the subsequent burning of the material, and the growth of reaction to detonation if strong shocks develop.

\subsection{Calculation of ignition without shocks}

The basis of our ignition criterion is the observation that in low-speed impacts, ignition is accompanied by significant shear deformation. We do not identify whether the localization mechanism is crystal twinning, continuum shear bands, friction, or grain-tograin slip. Instead, we use properties of the stress tensor to identify where shear deformation is occurring. The shear weighting factor, $f_{r}$, is given by

$$
f_{\tau}=\left(2-\frac{3\left|s_{2}\right|}{Y}\right),
$$

and has the value 2 in shear and the value 1 in compression or tension. When the factor is raised to a power, approximately 4 , there is an order of magnitude difference in the factor during shear deformation than in compression or tension. A similar factor uses the third invariant, $J_{3}$, of the stress-deviator tensor. The use of the third invariant, rather than the intermediate principal stress deviator is more customary in the solid mechanics literature. The similar, but not identical, factor would then be given by 


$$
f_{\tau}^{\prime}=\left(2-\frac{27\left|J_{3}\right|}{2 Y^{3}}\right)
$$

where

$$
J_{3}=s_{1} S_{2} S_{3}=\operatorname{det}\left(\mathbf{s}_{\mathbf{i j}}\right) .
$$

and $\mathbf{s}_{\mathrm{ij}}$ is the stress deviator tensor.

The ignition parameter also contains a weighting factor for the normal stress acting on the plane of maximum shear. When the normal stress is tensile, the weight is zero. This term was motivated by the view that more frictional work is done on an interface when the normal stress is more compressive. The ignition parameter is given by

$$
D_{\text {ignit }}=\int\left(2-\frac{27\left|J_{3}\right|}{2 Y^{3}}\right)^{5}\left(\frac{p+s_{2} / 2}{\sigma_{0}}\right)^{1 / 2} \dot{\varepsilon}_{P} d t .
$$

Here $\sigma_{0}$ is a parameter of order $50 \mathrm{MPa}$. Use of this criterion in simulations of the US and UK variants of the Steven test [19] have shown that ignition in both tests occurs when $D_{\text {ignit }}$ reaches 200 , although the peak pressures in the two tests differ by nearly a factor of 2. Similarly, scorch marks on the steel holder from UK tests are not on the axis of symmetry, where the pressure is largest, but at an intermediate radius, near where the criterion has its maximum value [20].

Computer simulations of Steven tests have shown that the value of the ignition criterion is quite sensitive to the coefficient of friction used [19, 20]. A separate parameter study, using a simplified geometry where a disk of explosive is crushed between two platens, illustrated both the sensitivity to friction, and how the localization of plastic strain depends on the pressure- and strain-rate- sensitivity of the flow stress. If the flow stress is assumed constant, the maximum strain developed, and the maximum value of the ignition criterion, is inversely proportional to the mesh size. With the pressure- and strain-rate dependence illustrated in Figs 2 and 3, the strain and ignition criteria decrease exponentially from the surface, where the e-folding distance is about $0.8 \mathrm{~mm}[18,19]$.

\subsection{Calculation of burning after ignition}

Once an element is ignited, it is assumed to burn as an assembly of particulates with a starting specific surface area. For smooth, compact objects the specific surface area is simply related to the size, D.

$$
\frac{S}{V}=\frac{6}{D}
$$

For spheres, $\mathrm{D}$ is the diameter. For cubes, $\mathrm{D}$ is the length of an edge. For naturally broken material, the specific surface area will be larger than that implied by its nominal size. However, the value depends on the scale of the irregularity that is resolved by the measurement technique.

For our purposes, the scale of irregularity is determined by the scale that can be sensed by an advancing flame. The frangibility test (shotgun test) [45] has been used to characterize propellant or explosive response to mechanical insult. Results of that test can be used to determine the specific surface area. The rate of change of the mass fraction converted to product for fragments burning from their surface inward is given by 


$$
\dot{\lambda}=\frac{S}{V} v_{b}(P)(1-\lambda)^{2 / 3},
$$

where $v_{b}$ is the pressure-dependent laminar burn speed. Here $S / V$ is the specific surface area before burning starts. At a given pressure, mass burning rate of broken material is larger than that measured for intact material. The ratio of the burning rates is the ratio of the surface areas. One complication in interpreting the test results is that fragmentation of the propellant or explosive is not uniform throughout the damaged volume. The pile of resulting fragments has a broad size distribution. We were, however, able to determine parameters for a simple linear dependence of specific surface area with plastic strain [9].

$$
\frac{S}{V}=A\left(\varepsilon_{p}-\varepsilon_{0}\right)
$$

where some critical value of plastic strain is required before fragmentation starts. A more physically based dependent variable would be plastic work, but for a given material they are related by the constitutive model for strength.

In HERMES we retained the dependence on plastic strain. We have chosen "reasonable" values for the two parameters, but there are no data. In principle, frangibility tests can be performed on explosives, and in practice they are sometimes tested [45]. In general, explosives are more difficult to ignite than propellants [45] so that the early pressure history recorded in these tests can be affected by the increased amount ignition aid needed to start the fragments burning. Recent observations of quasi-static damage [39] suggest that high confining pressure may significantly reduce the surface area developed with plastic strain. At present HERMES ignores this dependence.

\subsection{Calculation of the spread of an ignition front}

The permeation of hot product gas through a damaged reactant bed depends on the viscosity of the hot gas, the local channel dimension, and the local pressure gradient. Once hot gas arrives at the surface of cooler reactant, there is an induction time, which depends on the thermal properties of the reactant and the chemical kinetic reaction rates, before a self-sustaining local flame can start. This is an active research area for experiments and for multi-velocity, multi-phase computer simulations. Direct numerical simulation at the mesoscale, resolving both the boundary layer of flow through a channel, heat transfer between the hot gas and the solid, and the growth of reaction from a (reduced) chemical reaction net could be a fruitful and complementary approach. So far as we know, this has not yet been attempted.

In HERMES we have taken the expedient of using an input value for the ignition front velocity. We anticipate that experimental data and the results of multi-fluid simulations will be used to calculate the local time of ignition more adequately. In the interim, we have performed a limited number of parameter studies that illustrate that the ignition front velocity can have a significant effect on the subsequent reaction (Fig. 6) . Previous computer simulations with a much smaller sample volume showed less effect of ignition front velocity [17]. In those simulations, most of the porous bed had ignited before significant deformation occurred. 

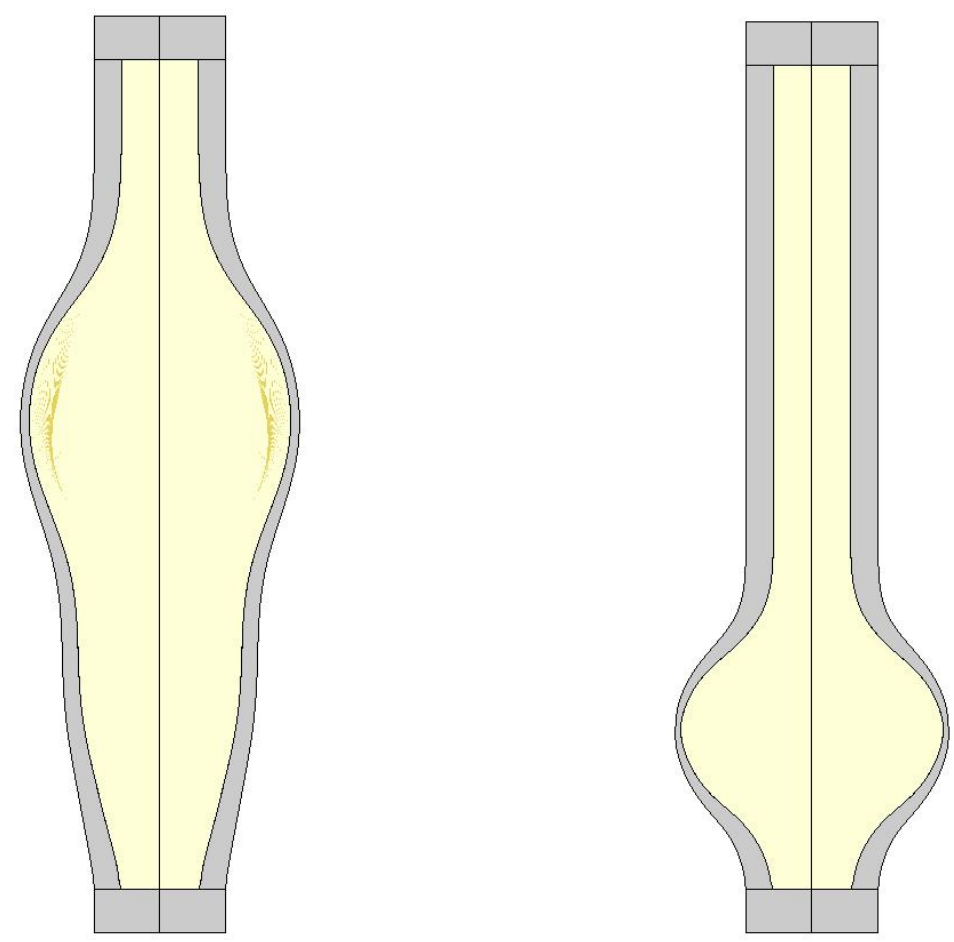

Figure 6. Calculation of a capped steel tube filled with 2-mm diameter explosive particles ignited at the bottom. The initial porosity is $20 \%$. The assumed ignition propagation speed from the ignited layer is 300 $\mathrm{m} / \mathrm{s}$ (left) and $30 \mathrm{~m} / \mathrm{s}$ (right). The deformation is shown at $1 \mathrm{~ms}$ after ignition (left) and $3.6 \mathrm{~ms}$ after ignition (right). The steel tubes are $48 \mathrm{~mm}$ OD, $28 \mathrm{~mm}$ ID, and $300 \mathrm{~mm}$ long. The endplates are $16 \mathrm{~mm}$ thick. The bottom endplate is constrained not to move vertically. The steel yield strength is $0.5 \mathrm{GPa}$, increasing to 0.7 $\mathrm{GPa}$ at 50\% (logarithmic) plastic strain

\subsection{Calculation of the growth to detonation}

If broken, porous explosive is shocked with sufficient amplitude, a detonation can develop. It is observed for all explosives that for one-dimensional plane shocks, the distance between the surface where a shock was introduced, and the point in the interior of the explosive where detonation develops (run-distance), depends on the shock amplitude. Weaker shocks require a longer run-distance to develop. Detonation models reproduce this feature. In addition, the CREST model [11-14, 30] distinguishes shocks from gradual pressure rises. We have chosen a simple subset of the CREST equations to couple detonation development with the growth of reaction due to burning in broken material. The subset of the CREST reaction model being used is given by 


$$
\begin{aligned}
& \dot{\lambda}=m_{2} \lambda_{2}(1-\lambda) \\
& \dot{\lambda}_{1}=\left(1-\lambda_{1}\right) \sqrt{-2 b_{1} \ln \left(1-\lambda_{1}\right)} \\
& \dot{\lambda}_{2}=\lambda_{1}\left(1-\lambda_{2}\right) \sqrt{2 b_{2}\left[\frac{b_{2} \lambda_{1}}{b_{1}}-\ln \left(1-\lambda_{2}\right)\right]} \\
& b_{1}=c_{0}\left(Z-c_{13}\right)^{c_{1}} \\
& b_{2}=c_{2}\left(Z-c_{13}\right)^{c_{3}} \\
& m_{2}=\frac{c_{10}}{\sqrt{b_{2}}}\left(Z-c_{13}\right)^{c_{11}}
\end{aligned}
$$

Here we have retained the notation of [11]. The $Z$ parameter is a function of entropy as described by $[11,30]$. The parameter $c_{13}$ may be positive, negative, or zero. The resulting pop plots (run distance as a function of shock pressure) exhibit either a cut-off shock pressure (below which a detonation does not form) when $c_{13}$ positive, or an asymptotic run distance approached at low shock pressure when $c_{13}$ negative, or a power-law dependence when $c_{13}$ zero.

In HERMES, the calculations (Eq. 47, 49) for the increase in mass fraction are integrated sequentially. The run-distance to detonation (pop-plot) for the UK explosive is shown in Fig. 7. Calculations were performed using Eq. 49 with $c_{13}$ zero to obtain the run distance to detonation. The parameter values were chosen to fit the normal density data. The calculated values at higher porosity are predictions.

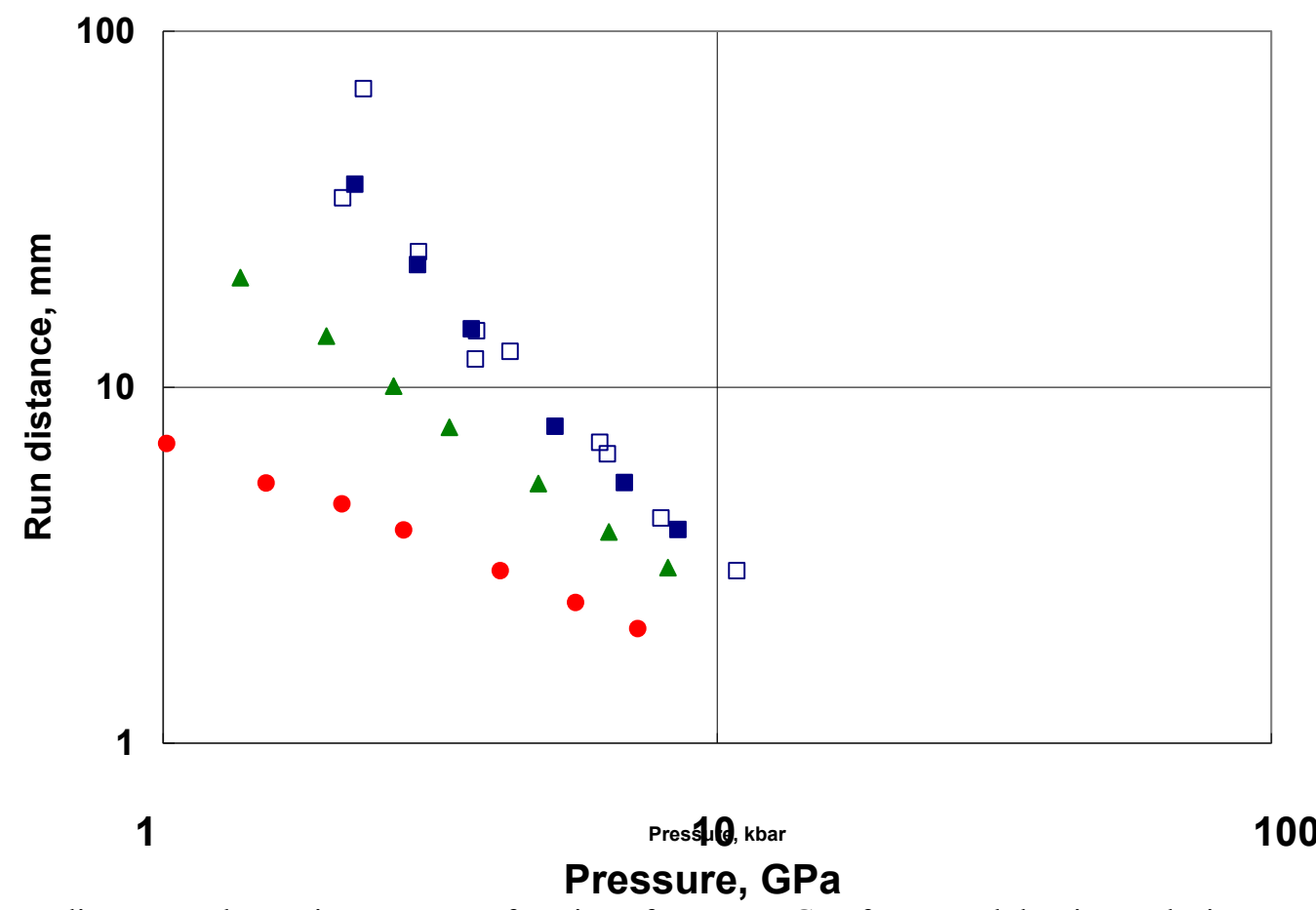

Figure 7. Run distance to detonation, $\mathrm{mm}$, as a function of pressure, $\mathrm{GPa}$ for normal density explosive (open squares) [14]; calculated run distance for normal density, which corresponds to $0.1 \%$ porosity (closed squares); $2.4 \%$ porosity (closed triangles); and $7.8 \%$ porosity (closed circles). 


\section{Calculation of porosity changes}

The porosity is changed in HERMES as a result of three phenomena: a reduction due to irreversible crush of the matrix, described in Section 2.5, an increase (dilatancy) due to the development of plastic volume strain when the strength depends on pressure, and an increase caused by an increase in the mass fraction of product (Eqs. 47, 49).

The increase in porosity due to dilatancy is given by

$$
\dot{\varphi}=\max \left[0, A y_{p} \dot{\varepsilon}_{p}(1-\varphi)\right],
$$

where the parameter $A$ varies between $A_{0}$ for small values of the dimensionless pressure derivative of the yield stress, $y_{p}$, and $A_{l}$ for large values of $y_{p}$.

$$
A=\frac{A_{0}+A_{1} y_{p}}{1+y_{p}} .
$$

The input parameters $A_{0}$ and $A_{1}$ are less than one, and we usually take $A_{0}$ to be zero. Taking the derivative of Eq. 28,

$$
y_{p i}=\frac{m / 2}{\sqrt{s+(m / 6)^{2}+m p /\left(R Y_{0}\right)}}
$$

for compression, $p>0$, and

$$
y_{p i}=\frac{3\left[\sqrt{s+(m / 6)^{2}}-m / 6-b_{\text {ten }}\right]}{b_{\text {ten }}}
$$

for tension $(p<0)$. From Eq. 25,

$$
y_{p r}=B_{r},
$$

when the residual strength is less than the intact strength. The value of $y_{p}$ is than

$$
y_{p}=y_{p i} f_{e}(1-\Omega)+y_{p r} \Omega .
$$

See Eqs. 22 and 23. When the Mohr-Coulomb model is chosen, the parameter $y_{p}$ is multiplied by the parameter $f_{\xi}$ (Eq. 41).

The increase in porosity due to burn is calculated by assuming the increase of burn fraction due to the growth of hot spots does not change the microscopic matrix pressure, $P_{m}$, where

$$
P_{m}=P_{M} / \Phi_{s} .
$$

After calculating a the new equilibrated pressure, the target macroscopic matrix pressure $P_{M t}$ is calculated by

$$
P_{M t}=P_{M} \frac{\Phi_{s}^{\text {new }}}{\Phi_{s}^{\text {old }}}
$$

where the volume fractions new and old refer to after and before the pressure is newly equilibrated. A value of $\alpha$ is calculated that is consistent with the target macroscopic matrix pressure and the matrix specific volume. The porosity $\varphi$ is increased consistently with the new volume fraction.

\section{Future developments}

As with any model, especially any relatively new model, there are many opportunities for improvement and development. These fall into three general and broad categories: algorithm improvements, new physics and chemistry submodels, and confirmation of functional forms and parameter values. The latter category includes both the applica- 
tion of mesoscale simulations and carefully designed and executed experiments. As noted by McAfee [46] in the context of DDT, the results of many experiments on energetic materials are difficult to interpret unambiguously. The advantage of simulations with HERMES and with mesoscale simulations is that the calculational results can help interpret the experimental results. In this section, we describe the three categories of developments, including specific examples where possible.

\subsection{Algorithmic improvements}

The algorithm used to calculate the rate-dependent yield strength (see the discussion in section 3 ) is slow to converge, typically requiring 20 iterations for every computational cycle. We found that a simple Newton solver would frequently not converge, as a result of discontinuous changes in slope to the yield strength, although when convergence was achieved, it was achieved quickly. Improvements here could significantly reduce the computational cost per cycle.

The calculation of pressure equilibrium with small amounts of product can be fragile, especially when the mesh becomes badly distorted. As a result the calculation is stopped with a "no convergence" message. A modest change in the element volume during a single computational step gives a very large change of volume to the gas, since it is more compressible. The net result is a bad estimate of the gas energy density, which can result in an unrealistically large or unrealistically low temperature. The resulting gas pressure is unsuited to achieving pressure equilibrium. Improvements to this algorithm are needed.

\subsection{Additional Physics and Chemistry submodels}

Tensile fracture is an additional way to create both porosity and surface area. In HERMES, this has been ignored, as it was for the PERMS model [9] that preceded it. For some mechanical insults, such as the Steven test, most of the material is under compression most of the time as it is deformed. As a result, ignoring tensile failure is a good approximation. For other insults, where there is less confinement, tensile failure may be a more important mechanism. We note that this is not a task undertaken lightly. Computer simulations of tensile fracture have had a long history of bad results. When the element fractures, it behaves like a stretched spring that is suddenly severed. The stored potential energy in the spring converts to kinetic energy. This sends unloading/reloading waves into the neighboring volume. If the spring is strong (large finite difference zone), neighboring zones can break due to the numerical "noise". The result is that very fine mesh resolution is required to develop a fracture pattern that is unchanged with further mesh refinement.

In general, the increase of porosity due to plastic strain (Eq. 50) should be manifest as an increase in pressure at constant volume. In this way, the unloading to zero pressure results in a volume larger than the starting volume. In our formulation, we have increased Herrmann's $\alpha$ parameter in step with the porosity. This approach, under various load paths needs, needs further testing and confirmation for simple rate-independent yield models, as well as our more complicated one. If the approach is found wanting, then the interplay of porosity and pressure must be reformulated.

The creation of specific surface area (Section 4.2) tacitly assumes that the volume of the finite element is sufficient to hold several of the fragments. Although the calculat- 
ed result is sensible for the case when the fragment is larger than the finite element, and variation with fragment size is smooth, it is inaccurate. Neighboring elements do not share a single large particle. More importantly, their delay before ignition should be set by the laminar burn speed, rather than by the propagation speed of an ignition front. The transition from large to small fragments, and the transition from ignition front propagation to laminar burn speed requires work. This is especially true when tensile fracture is implemented, because fragments created will be larger than a single volume element.

The present DDT model using CREST is appropriate for Type I DDT [46] where the initiation of detonation is the result of a sufficiently strong shock in under-dense explosive. This DDT type is observed for HMX explosives at low to moderate porosity (less than $40 \%$ porosity). For some other explosives, and at high porosity, Type II DDT can occur [46]. Small fragment size and large porosity result in a fast burn up for a relatively large volume of broken material. Our model can describe this feature, but does not describe the heterogeneous compaction observed for weak assemblies of highly porous material [46]. For the most part, the porosity that develops by mechanical damage is limited, and in our test simulations only grows large as a result of burn-up. As a result, this type II DDT may only occur in especially prepared porous beds rather than as a result of mechanical insult.

Similar considerations are required for XDT models, where the impact of a fragment field on a relatively rigid boundary can result in ignition that spreads rapidly. The fragment field may grow more dilute as a result of velocity gradients. In some instances subsequent impact of the fragments may result in similar response to Type I DDT and in other cases to ignition followed by Type II DDT. Applying the model to experimental situations in which XDT develops will help focus the development of an XDT capability for HERMES.

Present research in predicting the violence of thermal insults does not account for mechanical damage caused by differential thermal expansion in the build-up to cook-off, nor does it account for the extra mechanical damage caused by the local pressurization near the ignition site in the early stages of cook-off. To model this damage and the resulting violence requires coupling HERMES to heat transfer simulations and to incorporate a thermal ignition submodel.

\subsection{Assessing HERMES parameters and functional forms}

The HERMES model calculates the energetic response through the interaction of many physical and chemical processes, represented by interacting submodels. Although each of these submodels was developed to be consistent with our general experience in the behavior of explosives and propellants, the various submodels have not all been subject to the same level of vetting and parameter fitting. Throughout the text, we have indicated where additional data and/or insight would be of benefit, so that the predictions of various submodels could be subject to testing, not just the overall response. These predictions include strain and damage localization as influenced by friction, strength as influenced by lateral confinement and strain rate, the propagation of an ignition front through damaged explosive, and the effective specific surface area as sampled by the flame advancing into the fragments. 


\section{Summary}

We have developed a model to describe the mechanical and energetic response of propellants and explosives to mechanical insults. The model includes ignition from lowvelocity shear deformation, followed by more-or-less rapid burn of damaged material, as well as a simplified CREST detonation model for strong or developing shocks in damaged material. The HERMES model is based on those experimental observations that are available. Where data are missing, judgment is used to develop functional forms to predict the behavior.

We have indicated developments that would reduce the computer simulation time required by the model, and improve (or possibly confirm) the accuracy of the predictions. Extensions of the model include additional mechanical and thermo-mechanical mechanisms for damage, and for energetic response when damaged material is subsequently impacted. Future applications of the model include helping the design and interpretation of additional test vehicles to assess the adequacy of individual submodels, especially those developed in the absence of confirming data.

\section{Acknowledgements}

This work has been undertaken during three separate but related secondments to AWE. The work has been sponsored by four programs at LLNL, and in part by three programs at AWE. Leadership at LLNL has included Al Ross, Larry Fried, Bruce Watkins, Ron Streit, Keo Springer, Constantine Hrousis, Jon Maienschein, and Randy Simpson. Leadership at AWE has included Hugh James, Rod Drake, Andy Abbott, and Caroline Handley. I thank these and my many other colleagues at AWE and at LLNL who are not named here.

\section{References}

1. M. Wilkins, "Detonation and Shocks Review," Proceedings, Third Symposium on Detonation, Princeton University, NJ, September 26-29, 1960, p721.

2. M. Wilkins, J. French, R. Giroux, A computer program for calculating one-

dimensional hydrodynamic flow: KO code, Lawrence Radiation Laboratory report UCRL-6919, July 2, 1962.

3. M.L. Wilkins, "Calcul de Détonations Mono et Bidimensionnelles," Les Ondes de Détonation, Centre National de la Recherche Scientifique, 1962, p165.

4. M.L. Wilkins, in Methods in computational physics: advances in research and applications, Vol. 3: Fundamental methods in hydrodynamics, B. Alder, S. Fernbach, M. Rotenberg, eds. Academic Press, 1964.

5. J. von Neumann and R.D. Richtmyer, "A method for the numerical calculation of hydrodynamic shocks," J. Appl. Phys., 21, Mar. 1950, p232.

6. C.L. Mader, Numerical Modeling of Detonations, Univ. California Press, 1979, p207.

7. E.L. Lee and C. Tarver, "Phenomenological model of shock initiation in heterogeneous explosives," Phys. Fluids, 23, (12) Dec. 1980, p2362.

8. J.E. Reaugh, E.L. Lee, J.L. Maienschein, A.L. Nichols III, C.I. Merrill, R.R. Lambert, "Reduced Yield Detonation Characteristics in Large Failure Diameter Materials," Proceedings, $11^{\text {th }}$ International Detonation Symposium, Snowmass, CO, Aug 30-Sept 4, p1038, 1998. 
9. J.L. Maienschein, J.E. Reaugh, E.L. Lee, "Propellant Impact Risk Assessment Team Report: PERMS Model to describe Propellant Energetic Response to Mechanical Stimuli,” LLNL Report UCRL-ID-130077, Feb. 27, 1998.

10. M.R. Baer, C.A. Hall, R.L. Gustavsen, D.E. Hooks, S.A. Sheffield, G.T. Sutherland, "Isentropic compression Studies of the Mesoscale Response of Energetic Composites and Constituents," Proceedings, $13^{\text {th }}$ International Detonation Symposium, Norfolk, VA, p1070, July 23-28, 2006.

11. C.A. Handley, "The CREST reactive burn model," Proceedings, $13^{\text {th }}$ International Detonation Symposium, Norfolk, VA, p864, July 23-28, 2006.

12. N.J. Whitworth, C.A. Handley, and B.D. Lambourn, "Modelling Detonation Propagation and Failure in PBX9502 Using CREST," Proceedings, $14^{\text {th }}$ International Symposium on Detonation, Coeur d'Alene, ID, April 11-16, 2010, p61.

13. B.D. Lambourn, " The relation between reaction rate and shock strength: A possible second order improvement to the CREST reactive burn model," Proceedings of the Conference of the American Physical Society Topical Group on Shock Compression of Condensed Matter, Chicago, IL, 2011, in press.

14. C. A. Handley, "A comparison between entropy, temperature, and pressure-dependent reactive burn models," Proceedings of the Conference of the American Physical Society Topical Group on Shock Compression of Condensed Matter, Chicago, IL, 2011, in press. 15. J.E. Reaugh, Progress in model development to quantify High Explosive Violent Response (HEVR) to mechanical insult, Lawrence Livermore National Laboratory report LLNL-TR-405903, August 1, 2008.

16. J.E. Reaugh, Calculating the Dynamics of High Explosive Violent Response (HEVR) after Ignition, Lawrence Livermore National Laboratory report LLNL-TR-407915, October 20, 2008.

17. J.E. Reaugh, Implementation of Strength and Burn Models for Plastic-bonded Explosives and Propellants, Lawrence Livermore National Laboratory report LLNL-TR412938, May 2009.

18. J.E. Reaugh, Modifications and applications of the HERMES model: June-October 2010, Lawrence Livermore National Laboratory report LLNL-TR-462751, Nov 16, 2010. 19. J.E. Reaugh and A.G. Jones, "Mechanical Damage, Ignition, and Burn: Experiment, Model Development, and Computer Simulations to Study High-Explosive Violent Response (HEVR)," in Proceedings, $14^{\text {th }}$ International Symposium on Detonation, Coeur d'Alene, ID, April 2010, p909.

20. J.P. Curtis, A.G. Jones, C.T. Hughes, and J.E. Reaugh, "Modelling violent reaction following low speed impact on confined explosives," Proceedings of the Conference of the American Physical Society Topical Group on Shock Compression of Condensed Matter, Chicago, IL, 2011, in press.

21. J.W. Tringe, E.A. Glascoe, J.R. Kercher, R.M. Willey, H.K. Springer, D.W. Greenwood, J.D. Molitoris, L. Smilowitz, B.F. Henson, J.L. Maienschein, "Propagation of Reactions in Thermally-Damaged PBX-9501," in Proceedings, $14^{\text {th }}$ International Symposium on Detonation, Coeur d'Alene, ID, April 2010, p1010.

22. M.R. Baer, J.W. Nunziato, "A two-phase mixture theory for the deflagration-todetonation transition (DDT) in reactive granular materials," Int. J. Multiphase Flow, 12 (6) $1986, \mathrm{p} 861$. 
23. P.A. Gough, F.J. Zwarts, "Modeling Heterogeneous Two-Phase Reacting Flow," AIAA Journal, 17 (1) Jan, 1979, p17.

24. K.M. Roessig and J.C. Foster, Jr., "Experimental Simulations of Dynamic Stress Bridging in Plastic Bonded Explosives," Proceedings of the Conference of the American Physical Society Topical Group on Shock Compression of Condensed Matter, Atlanta, GA, June 24-29, 2001, p829.

25. A.W. Bishop, D.C. Webb, P.I. Lewin, "Undisturbed samples of London clay from Ashford common shaft - strength-effective stress relationships," Geotechnique, 15 (1) $1965, \mathrm{p} 1$.

26. J.E. Reaugh, Grain-scale Dynamics in Explosives, Lawrence Livermore National Laboratory report UCRL-ID-150388, 2002.

27. C.A. Handley, "Critical hotspots and flame propagation in HMX based explosives," Proceedings of the Conference of the American Physical Society Topical Group on Shock Compression of Condensed Matter, Chicago, IL, 2011, in press.

28. M. Cowperthwaite, "A Constitutive model for Calculating Chemical Energy Release Rates from the Flow Fields in Shocked Explosives," Proceedings, $7^{\text {th }}$ Symposium (International) on Detonation, Annapolis, MD, April 1981, p498.

29. J.E. Reaugh, E.L. Lee, "Isochoric Burn, an Internally Consistent Method for the Reactant to Product Transformation in Reactive Flow," Proceedings, $12^{\text {th }}$ International Detonation Symposium, San Diego, CA, g 11-16, 2002, p504.

30. B.D. Lambourn, "A complete EOS for non-reacted explosives," Proceedings of the Conference of the American Physical Society Topical Group on Shock Compression of Condensed Matter, Baltimore, MD, 2005, p165.

31. L.E. Fried, W.M. Howard, P.C. Souers, "EXP6: A new equation of state library for high-pressure thermochemistry," Proceedings, $12^{\text {th }}$ International Symposium on Detonation, San Diego, CA, August 11-16, 2002, p567.

32. S. Bastea, K. Glaesmann, and L.E. Fried, "Equations of state for high explosive detonation products with explicit polar and ionic species," Proceedings, $13^{\text {th }}$ International Detonation Symposium, Norfolk, VA, July 23-28, 2006, p1137.

33. W. Herrmann, "Constitutive Equation for the Dynamic Compaction of Ductile Porous Materials," J. Appl. Phys., 40 (6) May, 1969, p2490.

34. R.K. Linde, L. Seaman, D.N. Schmidt, "Shock response of porous copper, iron, tungsten, and polyurethane," J. Appl. Phys., 43, (8) 1972, p3367.

35. J.E. Reaugh, "Computer Simulations to study the explosive consolidation of powders into rods, ” J. Appl. Phys., 61 (3) 1987, p962.

36. O.Yu. Vorobiev, B.T. Liu, I.N. Lomov, T.H. Antoun, "Simulation of penetration into porous geologic media," Int. J. Impact Engineering, 34, April 2007, p721.

37. O. Vorobiev, "Generic strength model for dry jointed rock masses," International Journal of Plasticity, 24, p2221, 2008.

38. D.M. Williamson, C.R. Siviour, W.G. Proud, S.J.P. Palmer, R. Govier, K. Ellis, P. Blackwell, C. Leppard, "Temperature-time response of a polymer bonded explosive in compression," J. Phys. D.: Appl. Phys., 41, 2008, 085404.

39. D.A. Wiegand, B. Reddingius, K. Ellis, C. Leppard, "Pressure and friction dependent mechanical strength - cracks and plastic flow," Int. J. Solids and Structures, 48, June 2011, p1617. 
40. M.L. Wilkins, R. D. Streit, J. E. Reaugh, Cumulative-Strain-Damage Model of Ductile Fracture: Simulation and Prediction of Engineering Fracture Tests, Lawrence Livermore National Labs report UCRL-53058, Livermore, CA, October 3, 1983.

41 J.C. Jaeger, N.G.W. Cook, Fundamentals of Rock Mechanics, Chapman and Hall, 1979.

42. M.L. Wilkins, Computer Simulation of Dynamic Phenomena, Springer, 1999.

43. P.J. Rae and D.M. Dattelbaum, "The properties of poly(tetrafluoroethylene) (PTFE) in compression," Polymer, 45, 22004, p7615.

44. J.E. Reaugh, E.L. Lee, J.L. Maienschein, "The production of air blast from solid rocket motor fallbacks," JANAAF combustion meeting, 1997.

45. A.I. Atwood, K.P. Ford, D.T. Bui, P.O. Curran, T.M. Lule, "Assessment of mechanically induced damage in solid energetic materials, " $7^{\text {th }}$ Int. Symposium on Special Topics in Chemical Propulsion, Kyoto, Japan, September 2007.

46. J.M. McAfee, "The Deflagration-to-Detonation Transition," in B.W. Asay (ed.), Shock Wave Science and Technology Reference Library Vol. 5: Non-Shock Initiation of Explosives, Springer-Verlag, 2010, p483. 